\title{
The FAMU experiment at RIKEN RAL for a precise measure of the proton radius
}

\section{Bonesini ${ }^{* \dagger}$}

Sezione INFN Milano Bicocca, Dipartimento di Fisica G. Occhialini,

Università di Milano Bicocca, Italy

E-mail: maurizio.bonesini@mib.infn.it

The goal of the FAMU experiment at RIKEN RAL is to measure the hyperfine splitting of the ground state of the muonic hydrogen, to allow a determination of the proton Zemach radius with a precision better than $5 \times 10^{-3}$. The comparison of this measurement with the ones done with ordinary hydrogen may help to solve the so-called "proton radius puzzle", triggered by the $6 \sigma$ discrepancy in the proton charge radius value as extracted from muonic Lamb shift and the value based on e-p scattering and ordinary hydrogen spectroscopy.

European Physical Society Conference on High Energy Physics - EPS-HEP2019 -

10-17 July, 2019

Ghent, Belgium

*Speaker.

on behalf of the FAMU Collaboration 


\section{Introduction}

Many properties of the proton, such as its radius and anomalous magnetic moment, are not completely understood. The so-called proton radius "puzzle" [1] refers to the $6 \sigma$ discrepancy between the electron and muon determination of the proton charge radius. This discrepancy may be due to a violation of the electron-muon universality or simply to not well understood experimental systematic errors.

The FAMU (Fisica degli Atomi Muonici) experiment aims at the measurement of $\Delta E^{h f s}\left(\mu^{-} p\right)_{1 S}$ with a relative accuracy better than $10^{-5}[2,3,4]$. It makes use of a high intensity pulsed low-energy muon beam, stopping in a hydrogen target, to produce muonic hydrogen (in a mixture of singlet $\mathrm{F}=0$ and triplet $\mathrm{F}=1$ states) and a tunable mid-IR (MIR) pulsed high power laser to excite the hyperfine splitting (HFS) transition of the $1 \mathrm{~S}$ muonic hydrogen (from $\mathrm{F}=0$ to $\mathrm{F}=1$ states). Making use of the muon transfer from muonic hydrogen to another higher-Z gas in the target (such as $O_{2}$ or Ar), the $\left(\mu^{-} p\right)_{1 S}$ HFS transition will be recognized by an increase of the number of X-rays from the $\left(\mu Z^{*}\right)$ cascade, while tuning the laser frequency $v_{0}\left(\Delta E_{H F S}=h v_{0}\right)$. From the measure of $\Delta E^{h f s}\left(\mu^{-} p\right)_{1 S}$ the Zemach radius $r_{Z}$ of the proton may be deduced with a precision up to $5 \times 10^{-3}$, thus sheding new light on the problem of the proton radius puzzle.

The FAMU experiment is being performed in steps, starting from the study of the transfer rate from muonic hydrogen to another higher- $Z$ gas and ending with the full working setup with the pump MIR laser and a multipass optical cavity to determine the proton Zemach radius. The preliminary steps have allowed to determine the best mixture to be used inside the cryogenic target and optimize the operating conditions.

\section{Experimental layout at RIKEN-RAL}

The setup for the preliminary 2015-2016 (R582) FAMU data taking is schematically shown in figure 1 and fully described in reference [5]. It includes a beam hodoscope to characterize the impinging muon beam, a cryogenic target and detectors to see the characteristic low energy X-rays emitted in the process under study.

Data have been taken at the RIKEN-RAL muon facility[6], where the ISIS primary proton beam at $800 \mathrm{MeV} / \mathrm{c}$ produces a high-intensity pulsed muon beam, impinging on a secondary carbon target. The experiment makes use of a decay muon beam at $\sim 60 \mathrm{MeV} / \mathrm{c}$. The beam has a double pulse structure (70 ns FWHM widths, $320 \mathrm{~ns}$ peak to peak distance ) reflecting the primary beam structure. With an intensity of $\sim 8 \times 10^{4} \mu^{-} / s$ the beam has a momentum spread $\sigma_{p} / p=4 \%$ and an angular divergence $\sim 60 \mathrm{mrad}$.

\subsection{Beam characterization}

To monitor the beam parameters, avoid any undetected drift in beam shape and position and center the target a beam hodoscope system is needed. The beam hodoscope system has allowed to: tune the incoming beam, deliver timing information, as respect to radiofrequency, for DAQ readout and trigger, and monitor the intensity of each beam pulse. The system is based on three hodoscopes. Two of them are removable X/Y beam hodoscopes, made of 2 planes of $323 \times 3 \mathrm{~mm}^{2}$ square scintillating Bicron BCF12 fibers read at alternating fibers' ends by Hamamatsu S12572 

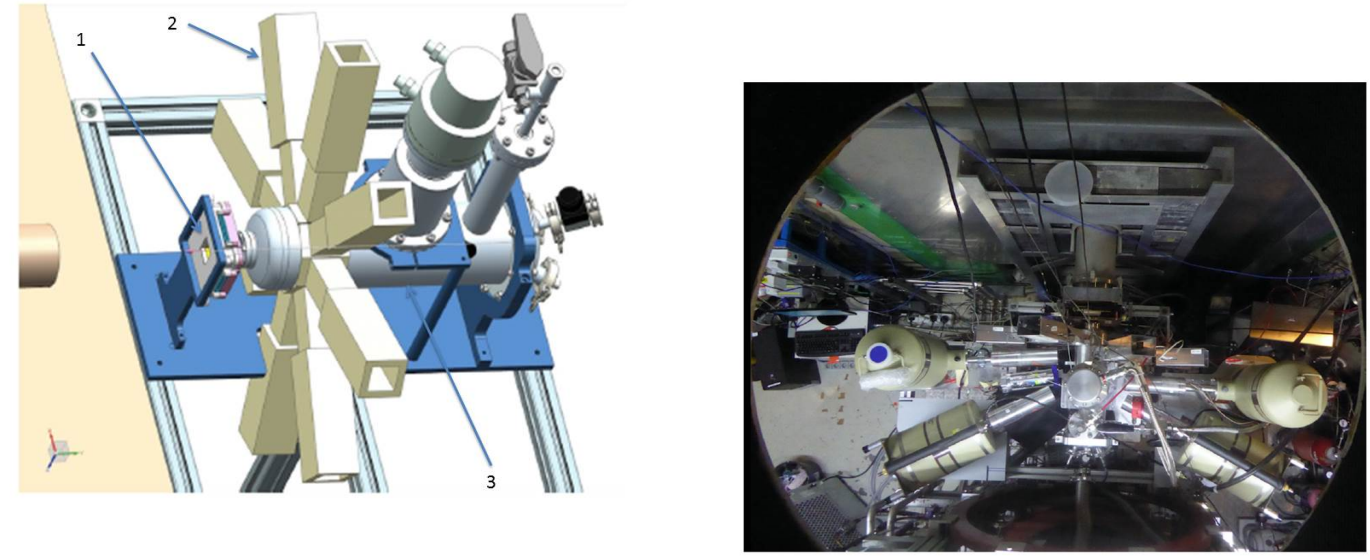

Figure 1: Left: layout used in the preliminary data taking of FAMU at RIKEN-RAL (R582). 1) is the $1 \mathrm{~mm}$ pitch beam hodoscope, 2) the crown of eight $\mathrm{LaBr}_{3}: \mathrm{Ce}$ detectors and 3) the cryogenic target. Right: picture of the experimental setup from the top. The four HpGe detectors are visible here.
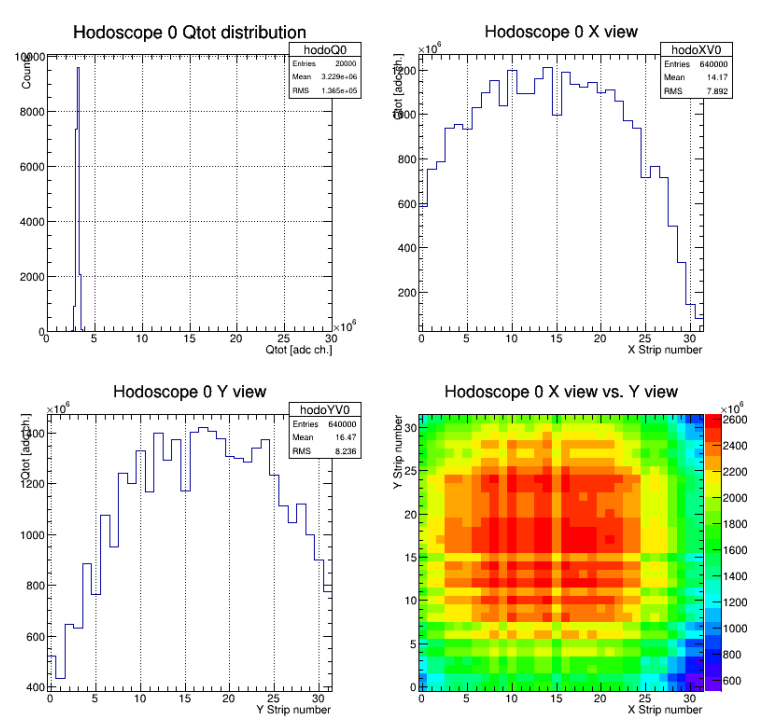

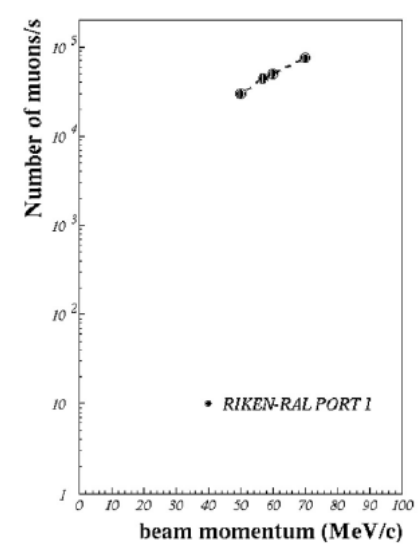

Figure 2: Left: from top to bottom, total deposited charge $\left(Q_{t o t}\right), \mathrm{X} / \mathrm{Y}$ beam projections and $\mathrm{X} / \mathrm{Y}$ beam profile for a $55 \mathrm{MeV} / \mathrm{c}$ beam momentum run. Right: beam intensity measured as a function of beam momentum.

silicon photomultiplier (SiPM), with $25 \mu \mathrm{m}$ cells [7, 8]. They have a $10 \times 10 \mathrm{~cm}^{2}$ active area. The third one makes use of $1 \mathrm{~mm}$ square BCF12 fibers, with white EMA coating, to ensure light tightness, read by $1 \mathrm{~mm}$ RGB Advansid SiPM, with $40 \mu \mathrm{m}$ cells $[8,9]$ and has an active area of $3.2 \times 3.2 \mathrm{~cm}^{2}$. It was permanently installed in front of the beam target window during data taking. As SiPM output signals are sizeable - $\sim 40 \mathrm{mV}$ with a S/B $\sim 10$ - no amplification stage is needed. Signals are digitized by CAEN V1742 FADCs ( $5 \mathrm{~Gb} / \mathrm{s}, 12$ bits, 1 Vpp dynamic range), providing information on timing, integrated charge and pulse height. The gain drift of SiPM with temperature will be controlled by CAEN DT5485 digital power supplies with built-in feedback on temperature, 
measured by Analog Devices TMP37 thermistors with a $\pm 2 \%$ precision. A X/Y beam profile, as measured at Port 1, is shown in the left panel of figure 2. A rms beam width less than $10 \mathrm{~mm}$ in both directions $(\mathrm{X}, \mathrm{Y})$ is measured. The total collected charge from the two planes of a detector $\left(Q_{t o t}\right)$ may be used, after a suitable calibration ${ }^{1}$, to estimate the incoming muon rate, as shown in the right panel of figure 2 .

To reduce the amount of material in front of the target a new hodoscope, based on $0.5 \times 0.5 \mathrm{~mm}^{2}$ square Bicron BCF12 scintillating fibers read by $1 \times 1 \mathrm{~mm}^{2}$ Hamamatsu S12751-050P SiPM will replace the current $1 \mathrm{~mm}$ pitch one in the final FAMU data taking. With $32+32 \mathrm{X}, \mathrm{Y}$ channels it will cover an active are of about $7.2 \times 7.2 \mathrm{~cm}^{2}$, to match the area of the new target entrance window.

\subsection{X-rays detection}

To extract the muonic X-ray lines of interest ( at $\sim 100-200 \mathrm{keV}$ ) with the best energy resolution and minimal events pile-up, hygroscopic $\mathrm{LaBr}_{3}: \mathrm{Ce}$ crystals were used ${ }^{2}$. In addition HpGe detectors (up to four were used at the same time) completed the experimental setup. They were used as a cross-reference due to their much better energy resolution. Their readout was performed through CAEN V1724 digitizers (14 bit, $100 \mathrm{MS} / \mathrm{s}, 10 \mathrm{Vpp}$ dynamic range). Detectors were placed around the region of maximal density of muonic hydrogen formation.

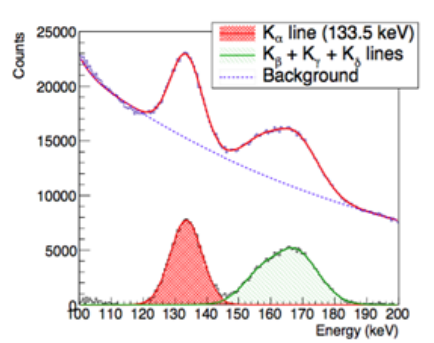

(a) $\mathrm{LaBr}_{3}$ counters spectrum

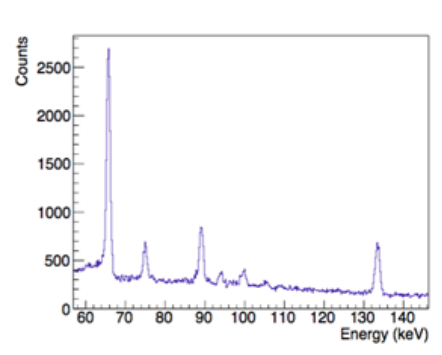

(b) HPGe spectrum

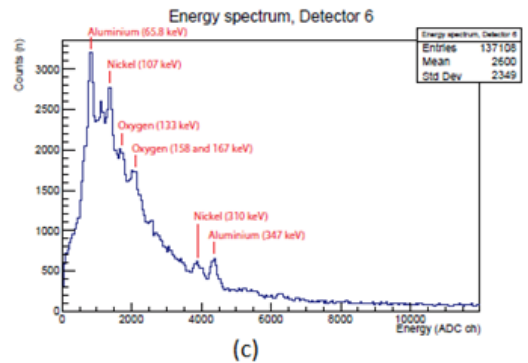

(c)

Figure 3: Typical muonic X-ray spectra recorded using (a) 1 " LaBr3:Ce counters, $K_{\beta}$ and $K_{\gamma}$ lines are not resolved; (b) the HpGe detectors in the same energy region and (c) 1/2" LaBr3:Ce counters with SiPM array readout.

The main X-rays detector is based on eight one inch diameter, one inch length $\mathrm{LaBr}_{3}: \mathrm{Ce}$ crystals arranged in a crown, read by Hamamatsu R112265-200 UBA 1" photomultipliers tube equipped with an active high voltage divider and a Digital Pulse Processor (DPP) [11]. Up to now, data have been acquired and processed by CAEN V1730 digiters (14 bit, $500 \mathrm{MS} / \mathrm{s}, 2$ Vpp dynamic range) in the framework of the general FAMU Data Acquisition System [12].

The collaboration has also developed a complementary read-out system for the $\mathrm{LaBr}$ :Ce crystals based on SiPM arrays. The advantage of the PMT readout as respect to SiPM readout of a better rise time ( $\sim 10 \mathrm{~ns}$ as compared to $20 \mathrm{~ns}$ ) is balanced, as regards the noise, by the use of smaller

\footnotetext{
${ }^{1}$ the calibration procedure makes use of laboratory test data taken with cosmic muons and tabulated values of $\mathrm{dE} / \mathrm{dx}$ vs momentum from PDG

${ }^{2}$ A preliminary study to asses if non-hygroscopic crystals, such as PrLuAg and Ce:GAAG, may be suitable was done and is reported in reference [10]
} 
crystal size: 1/2" instead of 1". Eight 1/2" LaBr with SiPM array readout have been recently tested and were used, due to their compact size, to instrument the most inaccessible regions around the target $[5,13,14]$. The readout was based on $4 \times 4$ array of $3 \times 3 \mathrm{~mm}^{2}$ Hamamatsu S13361 TSV SiPM. The output signals are summed up on a custom made PCB and digitized by CAEN V1730 digitizers. No amplification is needed, since the signal is about $100-200 \mathrm{mV}$.

\section{Experimental results on the transfer function}

The efficiency of the method proposed by the FAMU collaboration relies on the muon transfer rate dependence on the muonic hydrogen energy and on the epi-thermality of the muonic hydrogen at the moment of the muon transfer. In our investigation of the temperature dependence of the muon-transfer process from the thermalized $\mu \mathrm{p}$ atoms to oxygen, a strong monotonic rise of the transfer rate to oxygen in the temperature interval $103 \div 300 \mathrm{~K}$ has been observed [15], confirming previous experimental results at PSI [16]. Results on temperature dependence of the muon transfer rate to oxygen, as measured with $\mathrm{LaBr}_{3}: \mathrm{Ce}$ and $\mathrm{HpGe}$ detectors are shown in figure 4. Their theoretical interpretation is reported in reference [15], where a full discussion of systematics is also reported. Such a strong change enables to employ the muon transfer rate to oxygen as a signature of the kinetic-energy gain of the $\mu \mathrm{p}$ atom.

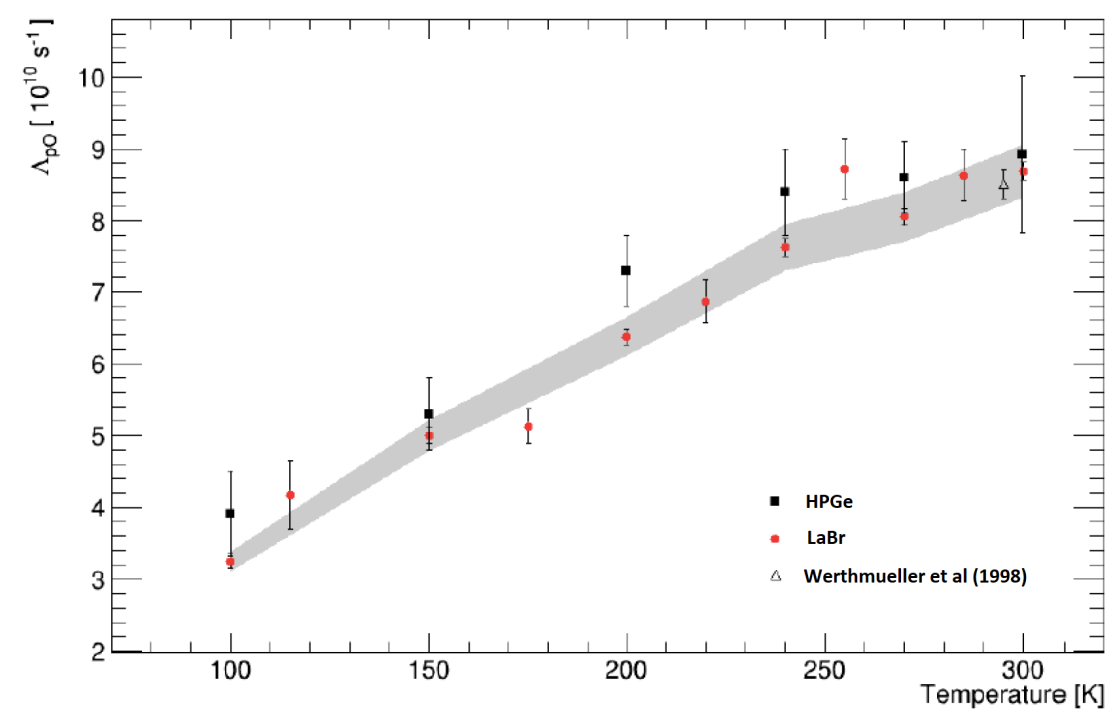

Figure 4: Temperature dependence of the muon transfer rate to oxygen as measured by FAMU independently with $\mathrm{HpGe}$ and $\mathrm{LaBr}$ detectors. Error bars indicate the statistical error only. The shaded region represents the systematic uncertainty of the result with $\mathrm{LaBr}_{3}: \mathrm{Ce}$. Data taken at PSI [16] are also reported.

\section{The final experiment layout and expected performances}

As the probability to stimulate an HFS transition is proportional to $W / \sqrt{(} T)$, with W laser pulse energy and $\mathrm{T}$ target temperature, the temperature should be the lowest possible to maximize 
the transition probability. At the same time, since a gas mixture of hydrogen and oxygen is used, the condensation temperature of oxygen limits the lowest possible temperature to about $60 \mathrm{~K}$.

In addition, to increase the interaction path between the $\mu^{-} \mathrm{p}$ atoms and the photons of the laser pulse an efficient multipass cavity is needed on the lines of what previously realized [17]. The optical cavity design is under study and presently encompass a $1.2 \times 1.2 \times 5 \mathrm{~cm}^{3}$ illuminated volume in the target, transverse to the impinging muon beam [18]. The optical path leading the laser light towards the internal optical cavity will be sensitive to vibrations. Therefore, the target cooling is based on a passive liquid nitrogen system to optimize mechanical stability and eliminate the vibrations produced by the cryogenic pumps. Figure 5 shows the actual target design, which has led to the choice of a liquid nitrogen $\left(\mathrm{LN}_{2}\right) 5$ liters reservoir, that will allow a duty cycle of about 5 days at the operating temperature of $80 \mathrm{~K}$.

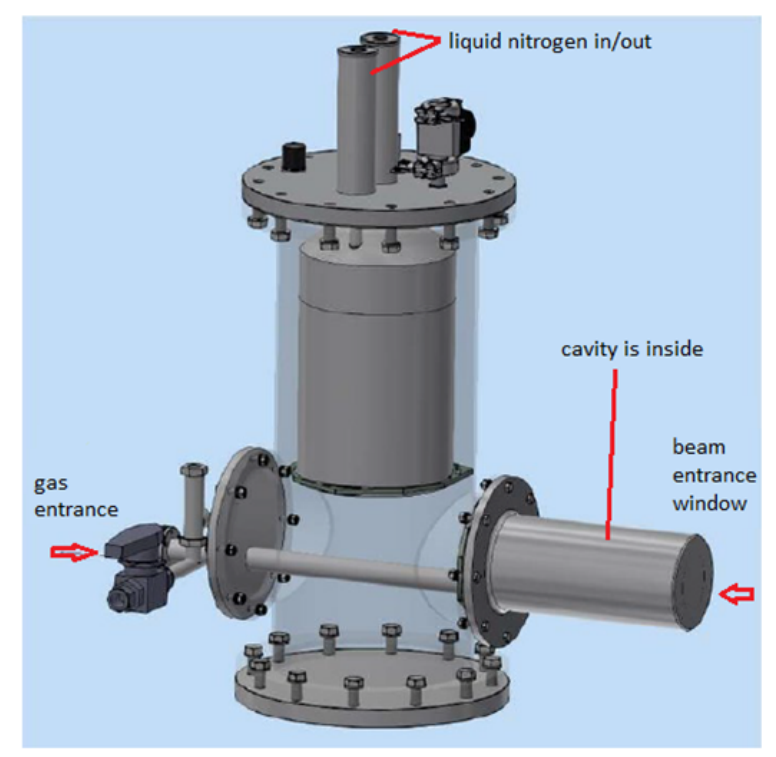

Figure 5: Current design the cryogenic gas target system. The optical cavity is not visible and is placed just behind the beam entrance window. Muon beam enters from the right.

The pulsed, tunable FAMU laser at $6785 \mathrm{~nm}$ has target parameters of an energy output of more than $1 \mathrm{~mJ}$, with a line width less than $0.07 \mathrm{~nm}^{3}$.

The chosen scheme is based on direct frequency generation (DFG) in LiInS2 crystals with pump and signal coming from one narrowband fixed wavelength Nd:Yag laser $(1064 \mathrm{~nm})$ and a tunable narrowband Cr:forsterite laser $(1262 \mathrm{~nm})$ pumped by another Nd:Yag laser, synchronized to the first one. For the generation of an energy greater than $1 \mathrm{~mJ}$ at $7 \mu \mathrm{m}$ the Nd:Yag laser must have an energy around $100 \mathrm{~mJ}$ and the Cr:forsterite of $35 \mathrm{~mJ}$. For details on the layout of the laser system see reference [19]. The laser wavelength $(\sim 6785 \mathrm{~nm})$ will be measured precisely $(\sim 30$ ppm) with solid state Fizeau interferometers and a cell filled with ${ }^{12} \mathrm{C}_{2} \mathrm{H}_{4}$.

From a detailed Montecarlo simulation the signal to background ratio may be estimated, optimizing the data taking conditions. For what concerns the target the optimal conditions require a

\footnotetext{
${ }^{3}$ pulse duration $10 \mathrm{~ns}$ at a $25 \mathrm{~Hz}$ repetition rate
} 
temperature of $80 \mathrm{~K}$, at a pressure of 7 bar for a $1 \%$ oxygen mixture. With the final laser energy of $\sim 4 \mathrm{~mJ}$ we expect a signal to background ratio around 10 in the selected time window. Background will be evaluated taking alternatively one beam spill with laser on and one with laser off.

\section{Conclusions}

The method proposed by the FAMU collaboration to measure the Zemach proton radius has been demonstrated feasible via our measurement of the transfer function to oxygen. The full experimental setup is under realization, including the optical cavity, the target and the DFG MIR laser. A first spectroscopic run is foreseen for the beginning of 2020, before the long ISIS 2021 shutdown.

\section{References}

[1] A. Antognini et al., Science 339, 417-420 (2013).

[2] D. Bakalov, E. Milotti, C. Rizzo, A. Vacchi and E. Zavattini, Phys. Lett. A172, 277-280 (1993).

[3] A. Adamczak, D. Bakalov, L. Stoychev, and A. Vacchi, Nucl. Instr. Meth. B281, 72-76 (2012).

[4] A. Adamczak et al., J. Instrum. 11, P05007 (2016).

[5] A.Adamczack et al., JINST 13 (2018) no 12, P12033

[6] T. Matsuzaki et al., Nucl. Instrum. Meth. A465 365-383 (2001).

[7] R. Carbone et al., JINST 10 (2015) no. 03,C03007

[8] M. Bonesini, et al., JINST 12 (2007) C03035

[9] M. Bonesini et al., Nucl. Instr.Meth. A936 (2019) 592; DOI: 10.1016/j.nima.2018.08.092

[10] M.Bonesini et al., Pos EPS-HEP2015 (2015) 244

[11] G. Baldazzi et al., JINST 12, C03067 (2017).

[12] M. Soldani et al.,Nucl. Instr. Meth. A936 327-328(2019); DOI: 10.1016/j.nima.2018.11.003

[13] M. Bonesini et al., PoS EPS-HEP2017 (2017) 777

[14] R. Benocci et al., Nucl. Instr.Meth. A936 570-571(2019); DOI: 10.1016/j.nima.2018.10.030

[15] E. Mocchiutti et al., submitted to Phys. Rev. A, ArXiv NuclEx/1905.02049

[16] A. Werthmuller et al., Hyperfine Interact. 116, 1-16 (1998).

[17] J. Vogelsang et al., Opt. Express 22, 13050-13062 (2014).

[18] D.Bakalov, M.Stoilov, EPJ Web of Conference 181,01033 (2018).

[19] L.I. Stoychev et al. in 9135, 91350J (International Society for Optics and Photonics, 2014). 